\title{
A density functional theory study of Raman modes of cadmium hexathiohypodiphosphate $\left(\mathrm{CdPS}_{3}\right)$
}

\author{
Abdul ShaKoor ${ }^{1 *}$, FAYYAz Hussain $^{1}$, NAJMUl Hassan $^{2}$, Abdul Majid $^{3}$, \\ Muhammad TARiQ BhatTi ${ }^{1}$, HaSSAN SidDiQue ${ }^{1}$ \\ ${ }^{1}$ Department of Physics. Bahauddin Zakariya University Multan, Pakistan, P.O.\#60800 \\ ${ }^{2}$ Physics Department, University of Hazara, Khyber Pukhtoon Khah Pakistan \\ ${ }^{3}$ Physics Department. University of Gujrat Punjab. Pakistan
}

\begin{abstract}
Raman scattering investigations based on density functional theory (DFT) calculations were performed to explore the vibrational modes of a cadmium hexathiohypodiphosphate $\mathrm{CdPS}_{3}$ single crystal. The calculations were performed to obtain the Raman spectra for the cadmium hexathiohypodiphosphate atoms to study the size dependence. Several vibrational modes indicating stretching and bending features related to $\mathrm{Cd}, \mathrm{S}$ and $\mathrm{P}$ atoms were observed. Modifications of the frequency and intensity of different Raman modes with an increase in the number of atoms in $\mathrm{CdPS}_{3}$ were discussed in detail. Hydrogen atoms were added in order to make the closed shell configuration and saturate the $\mathrm{CdPS}_{3}$ as per the requisite for calculating the Raman spectra. This produced some additional modes of vibration related to hydrogen atoms. Band gap and formation energy were also calculated. The results generated are found to be in close agreement with the experimental values.
\end{abstract}

Keywords: $\mathrm{CdPS}_{3}$; vibrational modes; Raman; density functional theory (DFT)

(C) Wroclaw University of Technology.

\section{Introduction}

Single crystal cadmium hexathiohypodiphosphates, here termed as $\mathrm{CdPS}_{3}$, have attracted much attention over the last thirty years because of their ability to form intercalation compounds with various inorganic and organic species [1]. The study of single crystal has been an interesting field of research for more than two decades. This is because it gives an opportunity to understand the physical properties in low dimensions and to explore their vast potential for application [2-6]. Over the past two decades research on quantum sized single crystal particles has increased enormously due to their novel applications in electronic and photonic devices [7-10]. The functionality of these devices has a strong correlation with the electronic and structural properties of the material used. These properties depend upon the vibrational characteristics of the material which are, in turn, related to the size

*E-mail: shakoor_47@bzu.edu.pk of single crystal and can be best explored using infrared and Raman scattering techniques [11-13]. $\mathrm{CdPS}_{3}$ is a promising candidate among IIB - VIA compound polymers due to its exceptional chemical and physical properties. It has been used as a window material for hetero junction solar cells to avoid the recombination of photo generated carriers, which improves the solar cells efficiency. $\mathrm{CdPS}_{3}$ quantum dots have also been deposited on substrates in solar cells to absorb sunlight and produce more free electrons to enhance the efficiency of solar cells. Insertion of intrinsic conducting polymer in layered host materials results in a dramatic modification of the physical properties, which has led to various applications as nonlinear optical materials [14, 15], magnetic materials [16, 17], catalysts and cathode materials in battery applications $[18,19]$. Different synthetic approaches [20, 21] combining a variety of host [22] and guest molecules [23-25] have been developed. A significant number of research groups are involved in synthesis and investigations of physical 
and chemical properties of $\mathrm{CdPS}_{3}$. The study of the size dependence of $\mathrm{CdPS}_{3}$ single crystal is an exciting research area since it provides tunable physical and chemical properties. However, few reports are available on the experimental study of vibrational dynamics of $\mathrm{CdPS}_{3}$ [26-28] and the literature on its counterpart using density functional theory based calculations is still lacking.

The structures of nanocrystal can be stabilized electrostatically by attaching a ligand with a negatively charged end group, thereby introducing repulsive force between the particles to keep them separated. For this purpose hydrogen may be used because it has an ambivalent nature. The study of hydrogen in crystalline semiconductors has been an active research area because of the ability of hydrogen to saturate the single crystals, passivate the surface states, terminate dangling bonds and remove other deleterious defects [29]. The presence of hydrogen in semiconductors is ambivalent, and it may, therefore, replace a cation or an anion, or form complexes with intrinsic impurities. Many groups have investigated the vibrational modes induced by hydrogen atoms in different semiconductors, like Si [30], ZnSe [22], GaAs [23, 24], etc. Although vibrational properties of hydrogenated III $-\mathrm{V}$ and IIB - VIA materials have been reported [25], no report is available in the literature on hydrogen in $\mathrm{CdPS}_{3}$ single crystals, to best of our knowledge. In this paper we report DFT based Raman spectroscopy to study the vibrational modes of hydrogenated $\mathrm{CdPS}_{3}$.

\section{Computational details}

All the calculations which are described in this work were DFT based and were performed in the Amsterdam density functional (ADF) program of version 2011.01 under local density approximation LDAVWN (Vosko-Wilk and Nausir). This code was used for the calculation of the number of atoms and dimension of elementary cell which was found to be composed of 32 atoms with dimension $6.21 \times 10.76 \times 6.86 \AA$. It was also exploited for generating the Raman spectrum of $\mathrm{CdPS}_{3}$. In the calculation, we distinguished the $\mathrm{Cd}$ $\left(1 \mathrm{~s}^{2}, 2 \mathrm{~s}^{2}, 2 \mathrm{p}^{6}, 3 \mathrm{~s}^{2}, 3 \mathrm{p}^{6}, 4 \mathrm{~s}^{2}, 3 \mathrm{~d}^{10}, 4 \mathrm{p}^{6}, 5 \mathrm{~s}^{2}, 4 \mathrm{~d}^{10}\right)$ $P\left(1 s^{2} 2 s^{2} 2 p^{6} 3 s^{2} 3 p^{3}\right)$ and $S\left(1 s^{2}, 2 s^{2}, 2 p^{6}, 3 s^{2}\right.$, $\left.3 \mathrm{p}^{4}\right)$ as core states, and $\mathrm{Cd}\left(5 \mathrm{~s}^{2}, 4 \mathrm{~d}^{10}\right)$ and $\mathrm{S}\left(3 \mathrm{~s}^{2}\right.$, $3 p^{4}$ ) as valence states. In order to provide electrostatic stabilization of the structures and saturation of the $\mathrm{CdPS}_{3}$ single crystal to complete the outer most shell, we used hydrogen, which is requisite for the calculation of Raman spectroscopy.

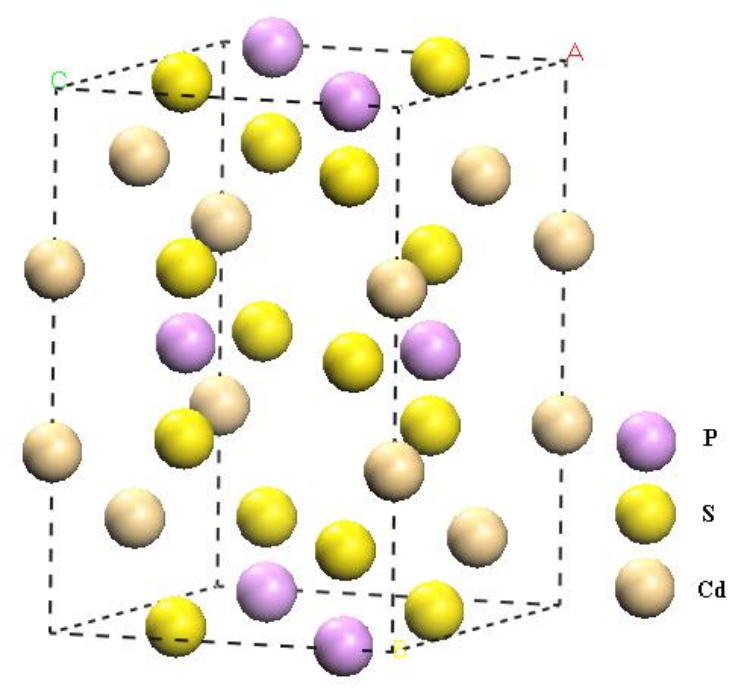

Fig. 1. The crystal structure of $\mathrm{CdPS}_{3}$.

\section{Experimental}

\subsection{Synthesis of $\mathrm{CdPS}_{3}$}

Single crystals $\mathrm{CdPS}_{3}$ were prepared by direct method by heating a mixture of stoichiometric amounts of elemental $\mathrm{Cd}, \mathrm{P}$ and $\mathrm{S}$ powders in an evacuated quartz tube at 800 to $900{ }^{\circ} \mathrm{C}$ for 10 days $[26,27]$. The resulting material contained mostly small crystals, but also included a few large, thin single crystals about $1 \mathrm{~cm}^{2}$ in area and 5 to $20 \mathrm{~mm}$ in thickness. Raman spectra for $633 \mathrm{~nm}$ exciting radiation were recorded on a Renishaw RM 1000 laser Raman (He-Ne laser) equipped with an Olympus metallurgical microscope and a CCD detector. The laser power at the sample was kept below $0.74 \mathrm{~mW}$ to avoid thermal degradation and the laser was shined for 5 seconds. The laser was focused by using a $50 \times$ objective lens and the spatial 
resolution was about $1 \mu \mathrm{m}$ [28]. The range of Raman spectra was of $400 \mathrm{~cm}^{-1}$ to $1000 \mathrm{~cm}^{-1}$.

\section{Results and discussion}

$\mathrm{A} \mathrm{CdPS}_{3}$ crystal structure is based on an unit cell containing three types of atoms. In particular, the tunneling current depends directly on the gap between the valence and conduction bands of the material [5]. As an electron energy increases, the electron density of states increases and more states become available for occupation. However, because there are no states available for electrons to occupy within the band gap, electrons at the conduction band edge must lose energy to enable transition to another available mode. In the present study the calculated band gap of $\mathrm{CdPS}_{3}$ is $3.38 \mathrm{eV}$, which is very close to the experimental value as shown in Fig. 2, and energy of formation is $-81.2332 \mathrm{eV}$. Due to the well-known deficiency of DFT method, the calculated band gap value is smaller than the experimental value of $3.51 \mathrm{eV}$ [6]; here we only compare the theoretical and experimental band gap values. It can be seen from the obtained band structure that the band gap of $\mathrm{CdPS}_{3}$ phase is slightly narrower between $\mathrm{Z}$ and $\mathrm{G}$ point. The valence band of $\mathrm{CdPS}_{3}$ is extended to lower energy level, while the conduction band is extended to higher energy level as shown in Fig. 2. The isolated $\mathrm{CdPS}_{3}$ sheet has a direct band gap at gamma point.

Table 1. Bond angle and bond length calculated using ADF.

\begin{tabular}{ccc}
\hline Atoms & $\begin{array}{c}\text { Bond length } \\
(\mathrm{pm})\end{array}$ & $\begin{array}{c}\text { Bond angle } \\
\left({ }^{\circ}\right)\end{array}$ \\
\hline \hline $\mathrm{P}(1)-\mathrm{P}(2)$ & 224.5 & \\
$\mathrm{P}(1)-\mathrm{P}(2)-\mathrm{S}(6)$ & & 34.6 \\
$\mathrm{~S}(5)-\mathrm{P}(1)$ & 203.2 & \\
$\mathrm{P}(1)-\mathrm{S}(8)-\mathrm{S}(5)$ & & 31.5 \\
$\mathrm{Cd}(17)-\mathrm{S}(6)$ & 271.2 & \\
$\mathrm{P}(2)-\mathrm{S}(69)-\mathrm{Cd}(17)$ & & 99.2 \\
$\mathrm{Cd}(17)-\mathrm{S}(16)-\mathrm{Cd}(20)$ & & 81.6 \\
\hline
\end{tabular}

The bond length and bond angles were also calculated and they are shown in Table 1.

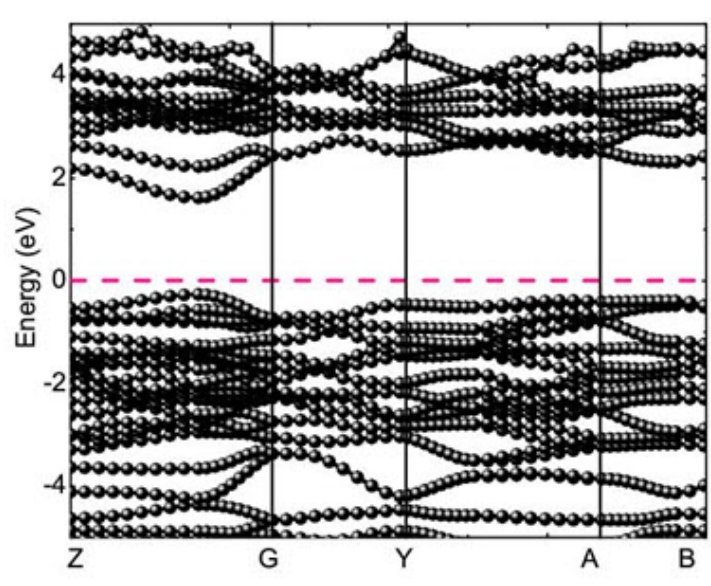

Fig. 2. Band structure of pristine CdPS3, red line shows the Fermi level.

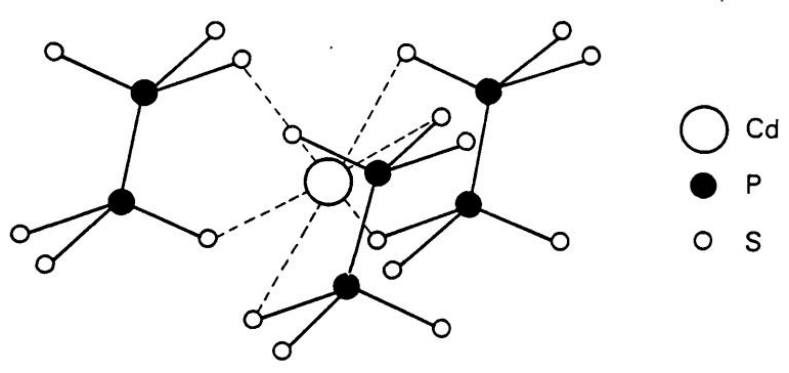

Fig. 3. Layered structure of $\mathrm{CdPS}_{3}$ [2].

\subsection{Experimental values}

Different structural and informative values of $\mathrm{CdPS}_{3}$ have been determined experimentally. The transition metal $\mathrm{CdPS}_{3}$ has a layered structure in which a molecular layer is made up of sheets of $\mathrm{Cd}$ and $\mathrm{P}$ atoms located between two sheets of $\mathrm{S}$ atoms, which is similar to that of transition metal dichalcogenides. Fig. 3 represents the layered structure of $\mathrm{CdPS}_{3}$ with the layer spacing of $6.55 \AA$. In the $\mathrm{CdPS}_{3}, \mathrm{Cd}$ atoms are half filled by $\mathrm{Cd}$ and half by P-P units, and the distortions lower the symmetry:

$\left(\mathrm{C}_{2} / \mathrm{m} ; \mathrm{a}=6.218(1), \mathrm{b}=10.763\right.$ (2) $\mathrm{c}=6.867$ (1) $\alpha, \beta=107.58$ (1); $\mathrm{z}=4$ ) [7]

All phosphorus atoms are crystallographically equivalent and occupy Wyckoff $4 \mathrm{i}$ positions. The sulfur atoms are of two types: one-third occupy another set of $4 \mathrm{i}$ sites, with the remainder in the $8 \mathrm{j}$ general positions [8]. 


\subsection{Raman spectra}

Fig. 4 shows the Raman spectra of cadmium hexathiohypodiphosphate $\left(\mathrm{CdPS}_{3}\right)$ single-crystal aided by DFT based calculations. Modifications in RS and in Raman intensity of different modes of vibrations are presented as a function of frequency. The DFT calculations have been reported to provide excellent vibrational frequencies [9]. Raman spectroscopy techniques combined with DFT calculations have been used as important tools to study molecules and biological compounds. The theoretical calculations could help in the interpretation of the experimental spectra [10].

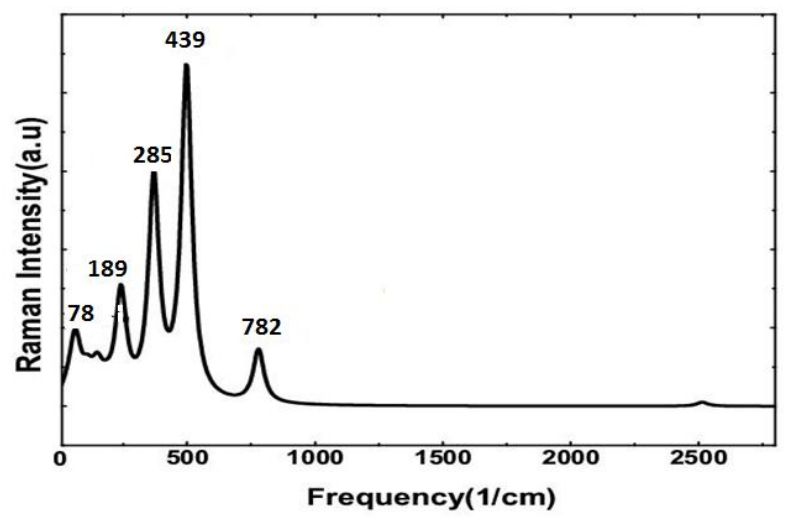

Fig. 4. Raman modes of $\mathrm{CdPS}_{3}$ obtained by ADF.

The calculated harmonic force constants and frequencies are usually higher than the corresponding experimental quantities, due to combination of electron correlation effects and basis set deficiencies. This is the reason to use a scaling factor for theoretical calculations. Nevertheless, after applying different scaling factors, the theoretical calculation reproduces the experimental data well. Certain values obtained by DFT methods are strongly underestimated. If the variations are omitted, the DFT calculations provide good linearity between the calculated and experimental frequencies. Similar information may be obtained from the study of oriented single crystals. However, the experience shows that the Raman emission from powdered crystalline solids is almost invariably depolarized as a result of the multiple reflections and refractions.
Fig. 5 shows the Raman spectra of cadmium hexathiohypodiphosphate $\left(\mathrm{CdPS}_{3}\right)$ single-crystal aided by DFT based calculations (Fig. 5a) and Raman spectra of $\mathrm{CdPS}_{3}$ obtained experimentally, using 633-nm exciting radiation. The laser was focused by using a $50 \times$ objective lens and the spatial resolution was about $1 \mu \mathrm{m}$. The range of Raman spectra shown in Fig. 5a and b is the same.

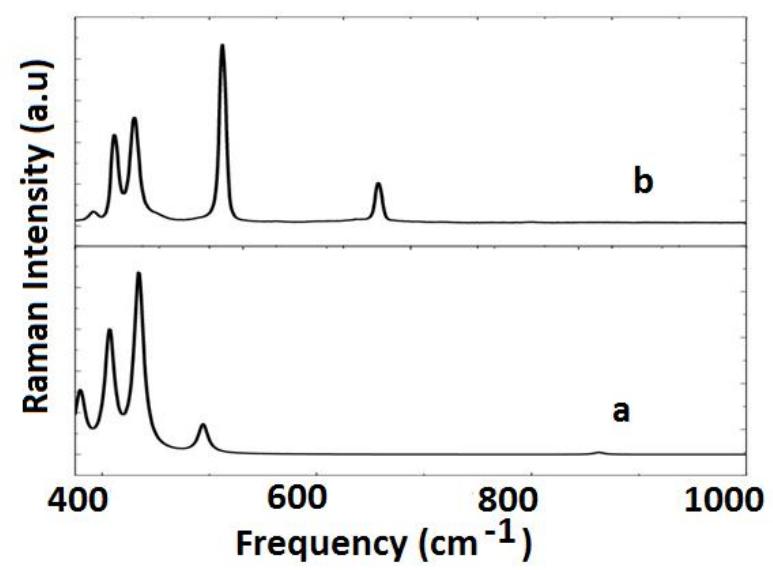

Fig. 5. Raman spectra obtained using (a) density functional theory (b) experimentally.

In Raman spectroscopy, the depolarization ratio is the intensity ratio between the perpendicular and parallel component of the Raman scattered light. Early work in this field was carried out by George Placzek, who developed the theoretical treatment of bond polarizability [12]. At frequency of $285 \mathrm{~cm}^{-1}$ Raman intensity becomes 49.31 and depolarization ratio is 0.0448 . All the Raman bands, frequency depolarization ratio and Raman intensity are shown in Table 2.

A fraction of the Raman scattered light has a polarization direction that is perpendicular to that of the incident light. This component is called the perpendicular component. Naturally, the component of the Raman scattered light whose polarization direction is parallel to that of the incident light, is called the parallel component, and the Raman scattered light consists of the parallel component and the perpendicular component. The ratio between these two components is expressed 
Table 2. Frequency depolarization ratio and Raman intensity.

\begin{tabular}{ccc}
\hline $\begin{array}{c}\text { Frequency } \\
\mathrm{cm}^{-1}\end{array}$ & $\begin{array}{c}\text { Depolarization ratio } \\
\text { (linear) }\end{array}$ & $\begin{array}{c}\text { Raman intensity (deg. not counted) } \\
\text { Angle }\left(^{\circ}\right) 4 / \mathrm{amu}\end{array}$ \\
\hline \hline 78.2877 & 0.474642 & 26.0243 \\
105.975 & 0.439829 & 16.3031 \\
134.668 & 0.695415 & 2.63614 \\
168.503 & 0.600209 & 4.33749 \\
184.548 & 0.428296 & 20.4120 \\
203.529 & 0.446043 & 15.8605 \\
228.006 & 0.731777 & 1.25866 \\
246.207 & 0.421406 & 1.62584 \\
285.067 & 0.448039 & 49.3134 \\
439.427 & 0.611817 & 19.0193 \\
527.911 & 0.170817 & 54.3850 \\
541.900 & 0.601128 & 22.3071 \\
782.020 & 0.740570 & 7.26032 \\
\hline
\end{tabular}

Table 3. Comparison of ADF and experimental values.

\begin{tabular}{ccccc}
\hline Atoms & $\begin{array}{c}\text { Bond length } \\
(\mathrm{pm})(\text { by ADF })\end{array}$ & $\begin{array}{c}\text { Bond angles } \\
\left(^{\circ}\right)(\text { by ADF })\end{array}$ & $\begin{array}{c}\text { Bond length } \\
(\mathrm{pm})(\text { experimental })\end{array}$ & $\begin{array}{c}\text { Bond angles } \\
\left({ }^{\circ}\right)\end{array}$ \\
\hline \hline $\mathrm{P}(1)-\mathrm{P}(2)$ & 224.5 & & 222.2 & 33.5 \\
$\mathrm{P}(1)-\mathrm{P}(2)-\mathrm{S}(6)$ & & 34.6 & & 31.4 \\
$\mathrm{~S}(5)-\mathrm{P}(1)$ & 203.2 & & 203.2 & \\
$\mathrm{P}(1)-\mathrm{S}(8)-\mathrm{S}(5)$ & & 31.5 & & 97.7 \\
$\mathrm{Cd}(17)-\mathrm{S}(6)$ & 271.2 & & 202.7 & 81.6 \\
$\mathrm{P}(2)-\mathrm{S}(69)-\mathrm{Cd}(17)$ & & 99.2 & & \\
$\mathrm{Cd}(17)-\mathrm{S}(16)-\mathrm{Cd}(20)$ & & 81.6 & & \\
\hline
\end{tabular}

as the depolarization ratio. As the frequency increases to $439 \mathrm{~cm}^{-1}$, the Ranman intensity and depolarizabilty ratio become 19.02 and 0.6118 , respectively. The two factors decrease as we increase the frequency. Under Placzek's polarizability approximation, it is known that the depolarization ratio of a totally symmetric vibrational mode is less than 0.75 , and that of the other modes equals to 0.75. A Raman band whose depolarization ratio is less than 0.75 is called a polarized band, and a band with a 0.75 depolarization ratio is called a depolarized band [13]. Raman modes at all the attained frequencies are polarized bands, as we observed that all depolarization ratios are less than 0.75 .

\subsection{Comparison of experimental and computational results}

A comparison between experimental and computational findings is very interesting and informative. The transition metal phosphorous trisulfides have a layered structure, where a molecular layer is made up of sheets of $\mathrm{Cd}$ and $\mathrm{P}$ atoms located between two sheets of $\mathrm{S}$ atoms, which is similar to that of transition metal dichalcogenides. $\mathrm{CdPS}_{3}$ has a layer spacing of $6.55 \AA$. The energy gap of $\mathrm{CdPS}_{3}$ crystal has been determined to be around $3.5 \mathrm{eV}$. Each $\mathrm{P}$ atom is 4-coordinated to three $\mathrm{S}$ and one $\mathrm{P}$ atom, with the bond lengths of 2.032 (2) 
and 2.027(1) Å to sulfur and 2.222 (2) $\AA$ to phosphorous. Detailed comparison is presented in the Table 3.

\section{Conclusions}

$\mathrm{CdPS}_{3}$ is a layered structure material. The ADF code was used to investigate its structure, formation energy and energy levels. It was found that the $\mathrm{P}-\mathrm{P}$ bond length was $224.5 \mathrm{pm}, \mathrm{S}-\mathrm{P}$ bond length was $203.2 \mathrm{pm}$ and $\mathrm{Cd}-\mathrm{S}$ bond length was $271.2 \mathrm{pm}$. Bond angles were $34.6^{\circ}$ for $\mathrm{P}(1)-\mathrm{P}(2)-\mathrm{S}(6), 31.5^{\circ}$ for $\mathrm{P}(1)-\mathrm{S}(8)-\mathrm{S}(5), 99.2^{\circ}$ for $\mathrm{P}(2)-\mathrm{S}(69)-\mathrm{Cd}(17)$ and $81.6^{\circ}$ for $\mathrm{Cd}(17)-\mathrm{S}(16)-\mathrm{Cd}(20)$. Raman modes were also observed by ADF. The theoretical peaks were very close to experimentally found modes. Slight differences were caused by theoretical approach of ADF. All the Raman modes were polarized as could be observed from depolarization ratio. The formation energy and energy gap was also very close to experimentally found data. All the results found from DFT strongly confirm successful completion of our theoretical approach.

\section{References}

[1] Zhukov V., Boucher F., Alemany P., Evain M., Alvarez S., Inorg. Chem., 34 (1995) 1163.

[2] Panina N., Demidov V. Simanova S., Rus. J. Chem., 78 (2008) 924.

[3] Venkataraman N., Vasudevan S., Proc. Indian Acad. Sci. Chem. Sci., 113 (2001), 347.

[4] Hemme W., Fujita W., Awaga K., Eckert H., Solid State Nucl. Mag., 39 (2011), 115.

[5] Park, C., Lee B., J. Korean Phys. Soc., 47 (2005), 665.

[6] Yang D., Westreich P., Frindt R., J. Solid State Chem., 166 (2002), 425.

[7] Siegler M., PARkin S., Brock C.P., Acta Crystallogr. B, 68 (2012), 400.

[8] Mo Y., Ong S.P., Ceder G., Phys. Rev. B, 84 (2011), 446.

[9] Jiang C.W., Zhou X., Xie R.H., Li F.L., Quantum Mater., 2 (2013), 363.

[10] Littleford R.E., TACKley D.R., Cherryman J.C., Dent G., Smith W.E., J. Mol. Struct., 692 (2004), 90.
[11] Zhukov V., Bowher F., Alemany P., Evain M., Alvarez S., Inorg. Chem., 34 (1995), 163.

[12] Placzek G., Teller E., Z. Phys. Chem., 81 (1933), 209.

[13] Furer V.L., Majoral J.P., Caminade A.M., KoVALENKo V.I., Vib. Spectrosc., 68 (2013), 70.

[14] Lagadic P.G., Lacroix P.G., Clément R., Chem. Mater., 9 (1997), 2012.

[15] LaCROIX P.G., Clément R., NAKATANi K., ZYSS J., Ledoux I., Science, 263 (1994), 660.

[16] Bérnard S., Léaustic A., Riviére E., Yu P., Clément R., Chem. Mater., 37 (2001), 3716.

[17] Floquet S., Salunke S., Boillot M.L., Clément R., VArret K., Boukheddaden F., RIVIÉRE E., Chem. Mater., 14 (2002), 4171.

[18] Brec R., Schleich D.M., Ouvrard G., Louisy A., RouXel J., Inorg. Chem., 18 (1979), 1814.

[19] Kerrache I., Julien C., Sourisseau C., Solid State Ionics, 92 (1996), 43.

[20] Jeevanandam P., Vasudevan S., Solid State Ionics, 104 (1997), 55.

[21] Clement R., Garnier O., Jegoudez J., Inorg. Chem., 25 (1986), 1409.

[22] Ouvard G., Brec R., Rouxel J., Mater. Res. Bull., 20 (1985), 1189.

[23] Qin J., Yang C., Yakushi K., Nakazawa Y., ICHIMURA K., Solid State Commun., 100 (1996), 431.

[24] Riede V., Neumann H., Sobotta H., Ascheron C., Novikov B., Solid State Commun., 61 (1987), 113.

[25] Mathey Y., Clement R., Sourisseau C., LuCAZEAU G., Inorg. Chem., 19 (1980), 2773.

[26] HaAs C., Horning D.F., J. Chem. Phys., 32 (1960), 1763.

[27] Shakoor A., Rizvi T.Z., J. Raman Spectrosc., 41 (2010), 240.

[28] Hourahine B., Jones R., Phys. Rev. B, 67 (2003), 205.

[29] Olea-Román D., Villeda-García J.C., Colorado-Peralta R., Solano-Peralta A., SAnchez M., Hernández-Ahuactzi I.F., CAstillo-Blum S.E., J. Mex. Chem. Society, 57 (3) (2013), 230.

[30] Bazian A., Taheri M., Alavi H., Rus. J. Gen. Chem.+, 84 (2014) 592. 